\title{
A REMARK ON THE ABSTRACT CAUCHY PROBLEM ON SPACES OF HÖLDER CONTINUOUS FUNCTIONS
}

\author{
MATTHIAS HIEBER * AND FRANK RÄBIGER
}

(Communicated by Palle E. T. Jorgensen)

\begin{abstract}
We prove that the generator of a $C_{0}$-semigroup on $C^{\alpha}\left(\mathbb{R}^{n}\right)$ is a bounded operator. Nevertheless, certain elliptic differential operators generate $\beta$-times integrated semigroups on $C^{\alpha}\left(\mathbb{R}^{n}\right)$ whenever $\beta>n / 2+1$.
\end{abstract}

\section{INTRODUCTION}

In 1985 it was proved by Lotz that the generator of a strongly continuous semigroup on a space of type $L^{\infty}(\mu)$ is a bounded operator (see [Na]). This means in particular that a given differential operator on $L^{\infty}(\mu)$ does not generate a strongly continuous semigroup on $L^{\infty}(\mu)$. Nevertheless, it was shown by Hieber [Hi2] that every elliptic differential operator $A$ with constant coefficients satisfying $\operatorname{Rep} \leq C(p$ denotes the symbol of $A)$ generates a $\beta$-times integrated semigroup on $L^{\infty}\left(\mathbb{R}^{n}\right)$ if $\beta>n / 2$.

In this note we prove similar results for the space $C^{\alpha}\left(\mathbb{R}^{n}\right)$, the space of all Hölder continuous functions on $\mathbb{R}^{n}$. In fact, the generator of a strongly continuous semigroup on $C^{\alpha}\left(\mathbb{R}^{n}\right)$ is a bounded operator and a differential operator of the above described kind generates a $\beta$-times integrated semigroup on $C^{\alpha}\left(\mathbb{R}^{n}\right)$ whenever $\beta>n / 2+1$.

\section{INTEGRATED SEMIGROUPS ON $C^{\alpha}\left(\mathbb{R}^{n}\right)$}

Let $0<\alpha<1$. We denote by $C^{\alpha}\left(\mathbb{R}^{n}\right)$ the space of all functions $f \in C\left(\mathbb{R}^{n}\right)$ such that

$$
\|f\|_{\Lambda_{\alpha}}:=\sup _{x \neq y} \frac{|f(x)-f(y)|}{|x-y|^{\alpha}}<\infty .
$$

$C^{\alpha}\left(\mathbb{R}^{n}\right)$ equipped with the norm $\|f\|_{C^{\alpha}}=\sup |f|+\|f\|_{\Lambda_{\alpha}}$ then becomes a Banach space. Peetre [P] considered the space $C^{\alpha}\left(\mathbb{R}^{n}\right)$ from the point of view of topological vector spaces and proved that $C^{\alpha}\left(\mathbb{R}^{n}\right) \cong l^{\infty}$ (see [P, p. 187]). This result has an important consequence in the theory of $C_{0}$-semigroups. Indeed, combining this result with the above-mentioned theorem due to Lotz we obtain the following.

Received by the editors December 5, 1990.

1991 Mathematics Subject Classification. Primary 34G10, 47D05.

* Supported by Deubche Forschungsgemeinschaft. 
Theorem 2.1. Let $(T(t))_{t \geq 0}$ be a $C_{0}$-semigroup on $C^{\alpha}\left(\mathbb{R}^{n}\right)$ with generator $A$. Then $A$ is a bounded operator.

Remark. The result remains true if $\mathbb{R}^{n}$ is replaced by the $n$-dimensional torus $T^{n}$ or by a bounded region $\Omega$ satisfying Peetre's condition (see [P, p. 188]).

Nevertheless, the theory of integrated semigroups enables us now to treat the Cauchy problem for certain differential operators on $C^{\alpha}\left(\mathbb{R}^{n}\right)$ in an elegant way. More precisely, let $A$ be a linear operator on a Banach space $E$ and let $\beta \geq 0$. We call the operator $A$ the generator of a $\beta$-times integrated semigroup $(S(t))_{t \geq 0}$ on $E$ if $(\omega, \infty) \subset \rho(A)$ (the resolvent set of $A$ ) for some $\omega \in \mathbb{R}$ and there exists a strongly continuous mapping $S:[0, \infty) \rightarrow L(E)$ satisfying $\|S(t)\| \leq M e^{\omega t} \quad(t \geq 0)$ for some $M \geq 0$ such that

$$
R(\lambda, A)=\lambda^{\beta} \int_{0}^{\infty} e^{-\lambda t} S(t) d t \quad(\lambda>\max \{\omega, 0\})
$$

In this case $(S(t))_{t \geq 0}$ is called the $\beta$-times integrated semigroup generated by $A$. In particular, a 0 -times integrated semigroup is a $C_{0}$-semigroup. The above integral is of course understood strongly in the sense of Bochner. For more information about integrated semigroups we refer to [A, $\mathrm{AK}, \mathrm{Hil}, \mathrm{KH}, \mathrm{Ne}$ ] and the references therein.

The following lemma is a straightforward modification of a theorem due to Arendt and Kellermann (see [AK, Proposition 3.1]). The proof is therefore omitted.

Lemma 2.2. Let $E$ be a Banach space and $A$ be a linear operator on $E$. Assume that there are constants $\omega \geq 0, M \geq 0$, and $\gamma \geq-1$ such that $\lambda \in \rho(A)$ for all $\lambda \in \mathbb{C}$ with $\operatorname{Re} \lambda>\omega$ and

$$
\|R(\lambda, A)\| \leq M|\lambda|^{\gamma} \quad(\operatorname{Re} \lambda>\omega) .
$$

Then the operator $A$ generates a $\beta$-times integrated semigroup on $E$ for all $\beta>\gamma+1$.

In order to prove that an elliptic differential operator $A$ with constant coefficients where the symbol $p$ of $A$ takes its values in a left-half plane generates an integrated semigroup on $C^{\alpha}\left(\mathbb{R}^{n}\right)$ we use a classical multiplier theorem for $C^{\alpha}$-spaces (see e.g., [T, p. 30, 93] or [M, Theorem I, p. 282]).

Lemma 2.3. Let $n \in \mathbb{N}, j=[n / 2]+1, M \geq 1$, and $m$ be a function of class $C^{j}$ on $\mathbb{R}^{n}$. If

$$
\left|\left(\frac{\partial}{\partial \xi}\right)^{k} m(\xi)\right| \leq\left(\frac{M}{1+|\xi|}\right)^{|k|} \quad(|k| \leq j),
$$

then there exists a constant $C$ such that for all $f \in C^{\alpha}\left(\mathbb{R}^{n}\right),\|\mathscr{F}-1(m \mathscr{F} f)\|_{C^{\alpha}\left(\mathbb{R}^{n}\right)}$ $\leq C M^{n / 2}\|f\|_{C^{\alpha}\left(\mathbb{R}^{n}\right)}$.

Here $\mathscr{F}$ denotes the Fourier transform in the sense of tempered distributions and $k$ a multiindex.

We now consider in detail elliptic differential operators with constant coefficients. If $p$ is the symbol of such an operator then $p$ is an elliptic polynomial 
on $\mathbb{R}^{n}$. In the following we always assume $\operatorname{Rep} \leq C$. We associate with $p$ a linear operator $A$ on $F:=C^{\alpha}\left(\mathbb{R}^{n}\right)$ as follows. Set

$$
D(A):=\left\{f \in F ; \mathscr{F}^{-1}(p \mathscr{F} f) \in F\right\}
$$

and define

$$
A f:=\mathscr{F}^{-1}(p \mathscr{F} f) \text { for all } f \in D(A) .
$$

Then the following holds.

Theorem 2.4. Let $p$ be an elliptic polynomial on $\mathbb{R}^{n}$ satisfying $\operatorname{Rep} \leq C$ for some constant $C$, and let $A$ be the operator on $C^{\alpha}\left(\mathbb{R}^{n}\right)$ defined as above. Then $A$ generates a $\beta$-times integrated semigroup $(S(t))_{t \geq 0}$ on $C^{\alpha}\left(\mathbb{R}^{n}\right)$ whenever $\beta>n / 2+1$.

Proof. We show that the resolvent $R(\lambda, A)$ of $A$ satisfies an estimate $\|R(\lambda, A)\|_{L\left(C^{\alpha}\left(\mathbb{R}^{n}\right)\right)} \leq M_{n}|\lambda|^{n / 2}$ for all $\lambda$ in a right-half plane. Then Lemma 2.2 yields the assertion.

To this end note first that the symbol $r_{\lambda}$ of $R(\lambda, A)$ is given by $r_{\lambda}(\xi):=$ $(\lambda-p(\xi))^{-1}$ for all $\xi \in \mathbb{R}^{n}$ and all $\lambda \in \mathbb{C}$ satisfying $\operatorname{Re} \lambda>C$. Moreover, we have $R(\lambda, A) f=\mathscr{F}^{-1}\left(r_{\lambda} \mathscr{F} f\right)$ for all $f \in F$ and all $\lambda \in \mathbb{C}$ satisfying $\operatorname{Re} \lambda>C$. Hence, by Lemma 2.2 it remains to show that for every $n \in \mathbb{N}$ there exists a constant $K_{n}$ such that

$$
\left|D^{k} r_{\lambda}(\xi)\right| \leq\left(\frac{K_{n}|\lambda|}{1+|\xi|}\right)^{|k|} \quad(|k| \leq j, \operatorname{Re} \lambda \geq C+1) .
$$

Let $C>0, \operatorname{Re} \lambda \geq C+1,|\xi|>1$, and $m$ be the degree of $p$. Then one can show by induction that for every multi-index $k \neq 0$ there exist constants $C_{1}, \ldots, C_{|k|}$ such that

$$
\left|D^{k} r_{\lambda}(\xi)\right| \leq \frac{C_{1}|\xi|^{m-|k|}}{|\lambda-p(\xi)|^{2}}+\frac{C_{2}|\xi|^{2 m-|k|}}{|\lambda-p(\xi)|^{3}}+\cdots+\frac{C_{|k|}|\xi|^{|k| m-|k|}}{|\lambda-p(\xi)|^{|k|+1}}
$$

for all $\lambda \in \mathbb{C}$ with $\operatorname{Re} \lambda \geq C+1$ and all $\xi$ with $|\xi|>1$. Therefore we obtain

$$
\left|D^{k} r_{\lambda}(\xi)\right|(1+|\xi|)^{|k|} \leq 2\left(\frac{C_{1}|\xi|^{m}}{|\lambda-p(\xi)|^{2}}+\frac{C_{2}|\xi|^{2 m}}{|\lambda-p(\xi)|^{3}}+\cdots+\frac{C_{|k|}|\xi|^{|k| m}}{|\lambda-p(\xi)|^{|k|+1}}\right)
$$

for all $\xi$ satisfying $|\xi|>1$. Since $p$ is elliptic there exist constants $K, L$ such that $|p(\xi)-\lambda| \geq K|\xi|^{m}$ for all $\xi \in \mathbb{R}^{n}$ satisfying $|\xi|>(|\lambda| / L)^{1 / m}=: L_{\lambda}$. Hence there is a constant $C_{k}^{\prime}$ such that

$$
\left|D^{k} r_{\lambda}(\xi)\right|(1+|\xi|)^{|k|} \leq C_{k}^{\prime} \quad(\operatorname{Re} \lambda \geq C+1)
$$

for all $\xi$ with $|\xi| \geq \max \left\{1, L_{\lambda}\right\}$. On the other hand, if $|\xi| \leq \max \left\{1, L_{\lambda}\right\}$, then it is clear that there is a constant $C_{k}^{\prime \prime}$ such that

$$
\left|D^{k} r_{\lambda}(\xi)\right|(1+|\xi|)^{|k|} \leq C_{k}^{\prime \prime}|\lambda|^{|k|}
$$

for all $\lambda \in \mathbb{C}$ with $\operatorname{Re} \lambda>C+1$. Choosing now $K_{n}:=\max \left\{C_{k}^{\prime}, C_{k}^{\prime \prime}\right\}$ we see that the estimate $(2.1)$ is fullfiled for all $\xi$ with $|\xi|>1$. Obviously such an estimate holds for all $\xi$ with $|\xi| \leq 1$. Thus Lemma 2.3 implies the existence of a constant $M_{n}$ such that

$$
\|R(\lambda, A)\|_{L\left(C^{\alpha}\left(\mathbb{R}^{n}\right)\right)} \leq M_{n}|\lambda|^{n / 2} \quad(\operatorname{Re} \lambda \geq C+1) .
$$


Remark. The order $\beta$ of integration in Theorem 2.4 can be improved considerably for the Laplacian $\Delta$ on $C^{\alpha}\left(\mathbb{R}^{n}\right)$. In fact, it follows from (2.2) that $\|R(\lambda, \Delta)\|_{L\left(C^{\alpha}\left(\mathbb{R}^{n}\right)\right)} \leq M_{n} /|\lambda|$ for all $\lambda \in \mathbb{C}$ with $\operatorname{Re} \lambda>0$. Therefore the Laplacian $\Delta$ generates a $\beta$-times integrated semigroup on $C^{\alpha}\left(\mathbb{R}^{n}\right)$ for all $\beta>0$.

\section{REFERENCES}

[A] W. Arendt, Vector valued Laplace transforms and Cauchy problems, Israel J. Math. 59 (1987), 327-352.

[AK] W. Arendt and H. Kellermann, Integrated solutions of Volterra integro-differential equations and applications, Volterra integrodifferential equations in Banach spaces and applications, (G. Da Prato and M. Iannelli, eds.), Proc. Conf. Trento 1987, Pitman Res. Notes in Math., vol. 190, Longman, Harlow, 1989, pp. 21-51.

[Hi1] M. Hieber, Laplace transforms and $\alpha$-times integrated semigroups, Forum Math. (to appear).

[Hi2] _ Integrated semigroups and differential operators on $L^{p}$ spaces, Math. Ann. (to appear).

[KH] H. Kellermann and M. Hieber, Integrated semigroups, J. Funct. Anal. 84 (1989), 160-180.

[M] A. Miyachi, On some singular Fourier multipliers, J. Fac. Sci. Univ. Tokyo Sect. IA Math. 28 (1981), 267-315.

[Na] R. Nagel (ed.), One-parameter semigroups of positive operators, Lecture Notes in Math., vol. 1184, Springer-Verlag, Berlin, Heidelberg, New York, Tokyo 1986.

[Ne] F. Neubrander, Integrated semigroups and their applications to the abstract Cauchy problem, Pacific J. Math. 135 (1988), 111-155.

[P] J. Peetre, New thoughts on Besov spaces, Duke Univ. Math. Ser. I, Duke Univ., Durham, 1976.

[T] H. Triebel, Spaces of Besov-Hardy-Sobolev type, Teubner Verlagsgesellschaft, Leipzig, 1978.

Georgetown University, Department of Mathematics, Washington, District of Columbia 20057

Mathematisches INSTitut der Universität, AUf Der Morgenstelle 10, D-7400 Tübingen, Federal RePUblic OF Germany 